\title{
Distance of three Supernova Remnants from H I line observations in a complex region: G114.3+0.3, G116.5+1.1, and CTB 1 $(\mathrm{G} 116.9+0.2)$
}

\author{
AYLİN YAR UYANIKER ${ }^{1,2}$, BÜLENT UYANIKER ${ }^{1,2}$, ROLAND KOTHES $^{1,3}$ \\ aylin@mpifr-bonn.mpg.de, uyaniker@mpifr-bonn.mpg.de, \\ roland.kothes@nrc-cnrc.gc.ca
}

\begin{abstract}
We present new radio continuum and $\mathrm{H}$ I images towards the supernova remnants (SNRs) G114.3+0.3, G116.5+1.1, and G116.9+0.2 (CTB 1) taken from the Canadian Galactic Plane Survey (CGPS). We discuss the dynamics of their $\mathrm{H}$ I environment and a possible relationship of these SNRs with each other. We discovered patches of $\mathrm{H}$ I emission surrounding G114.3+0.3 indicating a location in the Local arm at a distance of about $700 \mathrm{pc}$ in contrast to previous publications which proposed a Perseus arm location. The other two SNRs have radial velocities of $-17 \mathrm{~km} \mathrm{~s}^{-1}$ (G116.5+1.1) and $-27 \mathrm{~km} \mathrm{~s}^{-1}$ (CTB 1) according to related H I. However, the structure of the $\mathrm{H}$ I and its dynamics in velocity space suggest a possible relation between them, placing both remnants at a distance of about $1.6 \mathrm{kpc}$. CTB 1 appears to be embedded in an $\mathrm{H}$ I feature which is moving as a whole towards us with a velocity of about $10 \mathrm{~km} \mathrm{~s}^{-1}$. Furthermore, the off-centered location of CTB 1 in a large H I bubble indicates that the so-called breakout region of the remnant is in fact due to its expansion towards the low density interior of this bubble. We believe that the progenitor star of CTB 1 was an early B or O-type star shaping its environment with a strong stellar wind in which case it exploded in a Ib or Ic event.
\end{abstract}

Subject headings: supernova remnants, ISM: individual G114.3+0.3, G116.5+1.1, CTB $1(\mathrm{G} 116.9+0.2)$

\footnotetext{
${ }^{1}$ National Research Council, Herzberg Institute of Astrophysics, Dominion Radio Astrophysical Observatory, P.O. Box 248, Penticton, B.C., V2A 6J9 Canada

${ }^{2}$ Max-Planck-Institut für Radioastronomie, Auf dem Hügel 69, D-53121, Bonn, Germany

${ }^{3}$ Department of Physics and Astronomy, The University of Calgary, 2500 University Dr. NW, Calgary, $\mathrm{AB}, \mathrm{T} 2 \mathrm{~N} 1 \mathrm{~N} 4$ Canada
} 


\section{Introduction}

Towards Galactic Longitude $\ell \approx 115^{\circ}$, there are three extended, shell-type supernova remnants within an area of a few degrees; G114.3+0.3, G116.5+1.1, and G116.9+0.2 also known as CTB 1. The proximity of these sources and their relative sizes suggest a possible association. Kinematic distance estimates which are based on the correlation with surrounding atomic material are very difficult due to poor resolution of available data. H I absorption measurements fail because the sources are not bright enough to be absorbed by the warm foreground gas and possibly related $\mathrm{CO}$ was not detected. As a result distances to these remnants are poorly determined, especially for CTB 1, where the suggested values differ by more than a factor of two. In an attempt to improve the distance estimates of these remnants and to learn more about their environment we analyzed new $\mathrm{H}$ I observations from the CGPS (Taylor et al. 2003), which provides data at an unprecedented resolution of about $1^{\prime}$.

In this paper, we also present new radio continuum and polarization images of the region at $1420 \mathrm{MHz}$, from the CGPS database. A multi-frequency study of these remnants, especially of CTB 1, at 408, 2700, 4850 and $10500 \mathrm{MHz}$, including a comparative polarization analysis will be the topic of another publication (Yar-Uyanıker et al. 2003, in preparation).

In what follows we will review the characteristics of each individual source.

\subsection{G114.3+0.3}

G114.3+0.3 was identified as a supernova remnant by Reich \& Braunsfurth (1981) based on a comparison of radio continuum data at $11 \mathrm{~cm}$ and $21 \mathrm{~cm}$. They suggested a distance of $3.4 \mathrm{kpc}$ based on possibly related H I at a systemic velocity between $-35 \mathrm{~km} / \mathrm{s}$ and $-45 \mathrm{~km} / \mathrm{s}$. Fürst et al. (1993) and Kulkarni et al. (1993) associated this remnant with the pulsar PSR $2334+61$. The distance of the pulsar was obtained from its dispersion measure of $D M=55 \pm 10 \mathrm{pc} \mathrm{cm}^{-3}$ - now a more accurate value is available which gives $\mathrm{DM}=58.38 \pm 0.09$ (Mitra et al. 2003) - as 2.4(2.5) kpc, using the model of free electrons by Taylor \& Cordes (1993). The newly calculated model for the Galactic distribution of free electrons by Cordes \& Lazio (2002) is unfortunately not very useful for us since the authors incorporated a void in the direction of G114.3+0.3 most likely to accommodate for the larger distance to the SNR predicted by previous authors. As already discussed by Mitra et al. (2003), the rotation measure (RM) and dispersion measure of pulsars increase anomalously in the presence of small scale H II regions along the line of sight. They found that PSR $2334+61$ has an anomalous RM behaviour and the calculated foreground magnetic field along the line of 
sight is more than twice that of pulsars which are supposed to have no foreground object affecting their DM and RM values. Thus, the distance determination from the DM or RM of the pulsar may not be very accurate.

G114.3+0.3 has also been detected in optical emission (Fesen et al. 1997; Mavromatakis et al. 2002). Given the fact that optical emission suffers strongly from interstellar absorption the existence of optical emission from this SNR points to a rather close distance.

\section{2. $\mathrm{G} 116.5+1.1$}

G116.5+1.1 was also discovered and identified by Reich \& Braunsfurth (1981) at $21 \mathrm{~cm}$ and $11 \mathrm{~cm}$ wavelengths. Using low resolution data, from the Maryland-Green Bank survey (Westerhout \& Wendlandt 1982), Reich \& Braunsfurth (1981) noted a depression in H I between $-45 \mathrm{~km} \mathrm{~s}^{-1}$ and $-60 \mathrm{~km} \mathrm{~s}^{-1}$, which they translated to a distance between $3.6 \mathrm{kpc}$ and $5.2 \mathrm{kpc}$. They did not notice any other feature in their data which might be associated with this SNR. Fesen et al. (1997) have detected faint optical filaments in $\mathrm{H} \alpha$ and S II. The most prominent one, about $30^{\prime}$ long, positionally coincides with the bright radio rim.

\subsection{CTB $1(\mathrm{G} 116.9+0.2)$}

CTB 1 has been extensively studied in radio (Willis 1973; Dickel \& Willis 1980; Reich \& Braunsfurth 1981; Landecker et al. 1982) and in optical emission (van den Bergh et al. 1973; Lozinskaya 1980; Fesen et al. 1997). H $\alpha$ observations give a mean optical velocity of $v_{\mathrm{LSR}}=-33 \pm 2 \mathrm{~km} \mathrm{~s}^{-1}$ (Lozinskaya 1980). Reich \& Braunsfurth (1981) found a systemic velocity of $-35 \mathrm{~km} \mathrm{~s}^{-1}$ to $-50 \mathrm{~km} \mathrm{~s}^{-1}$, by relating the SNR to an H I structure partially encompassing the remnant from the south. However the resolution of their data $\left(\sim 10^{\prime}\right)$ does not allow a conclusive agreement between the remnant's shell and the H I filaments. They also mention the existence of an expanding atomic hydrogen shell associated with CTB 1, whose parts can be traced in the red and blue shifted components at velocities $v_{\mathrm{LSR}}=-20$ $\mathrm{km} \mathrm{s}^{-1}$ to $-30 \mathrm{~km} \mathrm{~s}^{-1}$ and $v_{\mathrm{LSR}}=-80 \mathrm{~km} \mathrm{~s}^{-1}$. However, the full extent of this shell,

expanding with a velocity of $20 \mathrm{~km} \mathrm{~s}^{-1}$ to $40 \mathrm{~km} \mathrm{~s}^{-1}$, remained undetermined due to the confusion with the local and outer arm emission.

Landecker et al. (1982) used H I data at 5' angular resolution, achieving a velocity resolution of $\sim 2.6 \mathrm{~km} \mathrm{~s}^{-1}$. Three major components in their spectra are evident, signaling the Local arm gas at -20 to $+20 \mathrm{~km} \mathrm{~s}^{-1}$, the Perseus arm gas at -30 to $-70 \mathrm{~km} \mathrm{~s}^{-1}$ and the Outer arm gas at -90 to $-110 \mathrm{~km} \mathrm{~s}^{-1}$. They did not find an exact correlation at any 
velocity. However, they found an area of $\mathrm{H}$ I emission between $-15 \mathrm{~km} \mathrm{~s}^{-1}$ and $-35 \mathrm{~km}$ $\mathrm{s}^{-1}$ which peaks at $-30 \mathrm{~km} \mathrm{~s}^{-1}$ and associated the remnant with this area. This velocity is also comparable to that obtained from the $\mathrm{H} \alpha$ data and implies a distance of $2.8 \mathrm{kpc}$ using a flat rotation curve with $R_{\odot}=8.5 \mathrm{kpc}$ and $v_{\odot}=220 \mathrm{~km} \mathrm{~s}^{-1}$ (Kerr \& Lynden-Bell 1986). Absorption measurements towards the western rim of CTB 1 provided no distance, because the foreground $\mathrm{H}$ I brightness $\left(\sim 25 \mathrm{~K} \mathrm{~T}_{\mathrm{B}}\right)$ is too high in comparison to the continuum emission ( $\sim 4 \mathrm{~K} \mathrm{~T}_{\mathrm{B}}$ with respect to the background). By comparing nearby open clusters, $\mathrm{O}$ associations, and H I regions Landecker et al. (1982) have concluded that CTB 1 must have a distance of $2.0 \pm 0.4 \mathrm{kpc}$. This would place the SNR in the interarm region between Local arm and Perseus arm.

According to Fich (1986) all three SNRs belong to a group of objects located within a large $\mathrm{H}$ I shell at a velocity of $v_{\mathrm{LSR}}=-46 \mathrm{~km} \mathrm{~s}^{-1}$, which translates to a distance of 4.2 kpc using a flat rotation curve. Fich (1986), on the other hand, quote the corresponding distance as $3.4 \mathrm{kpc}$, by comparing the velocity of the center of the Perseus arm with the distances of the H II regions in the neighboring area.

As demonstrated above, determining the distance of a supernova remnant is a difficult task. The established distances to many remnants are based on a relation between the surface brightness of the SNR to its diameter, usually called the $\Sigma-D$ relation. Since it has been shown that such a relation does not exist or just gives upper distance limits (see Green 1984; Berkhuijsen 1986, 1987, for a thorough discussion), we ignore previous distance estimates of these SNRs from the $\Sigma-D$ relations and concentrate on more direct observational evidence to determine distances; such as morphological and circumstantial coincidence of continuum and $\mathrm{H}$ I data, H I absorption features and relation of SNRs with the surrounding astronomical objects with known distances.

\section{Observations}

Radio continuum and $\mathrm{H}$ I line observations at $1420 \mathrm{MHz}$ were carried out simultaneously with the Synthesis Telescope of the Dominion Radio Astrophysical Observatory (DRAO) (Landecker et al. 2000) as part of the CGPS. The angular resolution of the final data products at this frequency is $49^{\prime \prime} \times 49^{\prime \prime} \operatorname{cosec}(\delta)$ for the radio continuum and $59^{\prime \prime} \times 59^{\prime \prime} \operatorname{cosec}(\delta)$ for the $\mathrm{H}$ I data. The $\mathrm{H}$ I data have a velocity resolution of $1.3 \mathrm{~km} \mathrm{~s}^{-1}$ covering 256 channels from $-158 \mathrm{~km} \mathrm{~s}^{-1}$ to $+52 \mathrm{~km} \mathrm{~s}^{-1}$ with a channel separation of $0.82 \mathrm{~km} \mathrm{~s}^{-1}$. The rms noise in the images at full resolution is $0.03 \mathrm{~K} \mathrm{~T}_{\mathrm{B}}$ in continuum and $3 \mathrm{~K} \mathrm{~T}_{\mathrm{B}}$ in $\mathrm{H} \mathrm{I}$. A detailed description of the data processing routines can be found in Willis (1999). Except the polarization maps, single antenna data are incorporated into the synthesis maps to ensure 
accurate representation of all structures up to the largest scales. The low spatial frequency H I data are from the Low Resolution DRAO Survey of the CGPS region observed with the DRAO 26-m Telescope (Higgs \& Tapping 2000).

\section{Results}

\subsection{Radio continuum emission}

In this section we briefly describe the structure of the radio continuum emission in the area around the three SNRs. All of the SNRs display partial shell structures placed on top of smooth emission plateaus. Fig. 1 displays a large region of approximately $5^{\circ} \times 3^{\circ}$ in size, which includes all of the SNRs discussed in this paper and shows their relative positions to each other. The contour map representations of these remnants, shown in Fig. 2 and 3, provide details about the surrounding emission plateaus.

CTB 1, at lower latitude in Fig. 3, is the smallest with an angular diameter of $38^{\prime} \times 32^{\prime}$ and has the highest surface brightness. Its emission is concentrated in two bright arcs in the south-east and in the west; and these two arcs, apart from a region with less emission (the breakout region) towards north-east, form an almost circular shape.

G114.3+0.3 (Fig. 2) shows a dominant thin arc in the south-west. Within its boundary is the bright $\mathrm{H}$ II region $\mathrm{S}$ 165, which is not related to the supernova remnant (Reich \& Braunsfurth 1981). The angular size of this SNR is $82^{\prime} \times 65^{\prime}$. The associated pulsar PSR $2334+61$ is located at the center of the remnant (Fürst et al. 1993; Kulkarni et al. 1993; Becker et al. 1996).

G116.5+1.1 (Fig. 3) is located just north of CTB 1, and has an angular size of $81^{\prime} \times 62^{\prime}$. The strongest emission from the remnant is concentrated in a relatively thick arc, $\sim 18^{\prime}$ wide, at the opposite side of CTB 1. A smooth emission plateau with a brightness of $0.5 \mathrm{~K}$ $\mathrm{T}_{\mathrm{B}}$, which is encircling the two SNRs, is placed on top of the smooth Galactic background

component of about $5 \mathrm{~K} \mathrm{~T}_{\mathrm{B}}$, in this direction. This emission plateau might indicate a possible association.

\subsection{Polarized emission}

Polarization properties of the DRAO Synthesis Telescope are given in Taylor et al. (2003) and preparation of polarized survey images are discussed in detail by Uyanıker et al. (2003). Portions of the polarization data relevant to this paper, showing all three remnants, 
are plotted in Fig. 4 at $2^{\prime}$ resolution. Polarized emission above $0.1 \mathrm{~K} \mathrm{~T}$ is detected from both G114.3 and G116.5. They appear as partial shells with fine structure in polarization. CTB 1, on the other hand, appears to be weaker.

In the following sections we will use the polarization data as an argument to claim a close distance for the SNRs. According to Uyanıker et al. (2003) there exists a maximum distance from which the polarized emission reaches us without being totally depolarized. This distance depends on the electron density, the strength of the magnetic field and the distance from which the emission originates. Uyanıker et al. (2003) called this distance the "polarization horizon". Clearly the horizon is not single valued and varies with the wavelength of the observation and physical parameters along the line of sight. This, however, implies a strong relationship between the detectability of the polarized emission and the distance of the object.

A phase shift of $90^{\circ}$ between two polarization vectors would cause total depolarization. To achieve this phase shift a rotation measure of $36 \mathrm{rad} \mathrm{m}^{-2}$ at $21 \mathrm{~cm}$ is required. Towards the SNRs the magnetic field strength parallel to the line of sight is $\mathrm{B}_{\|}=1 \mu \mathrm{G}$ (Mitra et al. 2003). This yields a dispersion measure of $44 \mathrm{~cm}^{-3}$ pc according to Eqn. 1, for which the electron density model of Taylor \& Cordes (1993) gives a distance of $2.2 \mathrm{kpc}$ in this direction. This value is similar to the one found by Uyanıker et al. (2003) towards $82^{\circ}<\ell<95^{\circ}$ and implies that rotation measure sets an upper limit on the path length of the observable polarized emission, namely the distance of the object.

\subsection{Neutral Hydrogen}

The $21 \mathrm{~cm}$ line data from the CGPS reveal new information about the kinematics of the neutral hydrogen surrounding these three SNRs. Figures 5 and 6 present averaged H I maps towards these SNRs.

Bright and large H I emission patches surround G114.3+0.3, centered at a velocity of $v_{\mathrm{LSR}}=-6.5 \mathrm{~km} \mathrm{~s}^{-1}$ (see Fig. 5). No other $\mathrm{H}$ I feature which shows any resemblance to the SNR can be found in the entire H I data cube. Using a flat rotation model for the Milky Way with $R_{\odot}=8.5 \mathrm{kpc}$ and $v_{\odot}=220 \mathrm{~km} \mathrm{~s}^{-1}$ this would translate to a kinematic distance of $700 \mathrm{pc}$, which is significantly closer than all previously published distance estimates all of which proposed a location within Perseus arm. The structure of the H I emission does not indicate that the environment was shaped by the stellar wind of the progenitor star, in which case we would expect a more dynamic environment dominated by thin filaments.

The H I environment of G116.5+1.1 as displayed in Fig. 6 (left panel) looks like it was 
carved out by the expanding shockwave of the supernova explosion. To the west and south there are bright patches of $\mathrm{H}$ I following nicely the curvature of the radio bright shell. To the east most of the area shows a lack of H I. Like for G114.3+0.3 it looks like the ambient medium was not influenced by stellar wind effects of the progenitor star. The central radial velocity of the $\mathrm{H} \mathrm{I}$ structure is about $-17 \mathrm{~km} \mathrm{~s}^{-1}$ which translates to a kinematic distance of about $1.6 \mathrm{kpc}$.

CTB 1, on the other hand, is surrounded by an "open-end-ring" of neutral hydrogen (see Fig. 6, right panel) centered at $v_{\mathrm{LSR}}=-27 \mathrm{~km} \mathrm{~s}^{-1}$, which translates to a kinematic distance of $2.5 \mathrm{kpc}$. The shape of the surrounding H I ring coincides well with the breakout appearance of the SNR's radio continuum emission as seen in Fig. 3. In this case the structure of the surrounding H I looks more dynamic. Here we find thin filaments and shells indicating a highly dynamic environment in contrast to the smooth bright patches surrounding the other SNRs (better presented in Figs 8-9). The progenitor star of CTB 1 obviously had a strong stellar wind and affected the ambient medium significantly.

In the area around CTB 1 the local arm and the Perseus arm are very distinct in velocity space as clearly demonstrated in the velocity-distance diagram in Fig. 7, where we plotted SNRs and H II regions within $20^{\circ}$ of Galactic longitude of the SNRs as a function of distance to emphasize this fact. Local objects have radial velocities above $-20 \mathrm{~km} \mathrm{~s}^{-1}$ while Perseus arm sources show radial velocities below $-30 \mathrm{~km} \mathrm{~s}^{-1}$. The decision whether G114.3 and G116.5 reside in the local arm or the Perseus arm, based on the radial velocities of $-6.5 \mathrm{~km}$ $\mathrm{s}^{-1}$ and $-17 \mathrm{~km} \mathrm{~s}^{-1}$ respectively, is made very simple. However, the associated H I shell of CTB 1 with a radial velocity of $-27 \mathrm{~km} \mathrm{~s}^{-1}$ indicates an interarm location close to Perseus arm. Because of the complex and highly dynamic environment of the SNR, which suggests a highly active progenitor star with a strong stellar wind, we believe that CTB 1 must reside in one of the spiral arms. This makes CTB 1 either a blueshifted local or a slightly redshifted Perseus arm object.

\section{Discussion}

\subsection{G114.3+0.3}

Besides the possibly related H I structure we found, a fact that most of the time is somewhat subjective anyway, we have many other observational and circumstantial evidence which can give us constraints on the distance of this SNR. First of all, optical filaments were detected by Fesen et al. (1997) and Mavromatakis et al. (2002). Since optical emission suffers from interstellar absorption its presence indicates a rather low distance. In the direction of 
G114.3+0.3 this would mean that a Perseus arm location is rather unlikely. This fact is supported by the presence of polarized emission in the CGPS at $21 \mathrm{~cm}$ (see Fig. 4) which indicates a distance in front of the so-called polarization horizon, which was found to be at about $2 \mathrm{kpc}$ for CGPS data (Uyanıker et al. 2003). These two constraints already imply a location in the Local arm rather than the Perseus arm. An additional upper limit can be derived from the distance to $\mathrm{S} 165$ which is not depolarizing the $11 \mathrm{~cm}$ continuum emission at its line of sight and hence must be located behind the SNR (Reich \& Braunsfurth 1981). A new spectroscopic distance estimate (Brand \& Blitz 1993) puts this H II region at a distance of $1.6 \mathrm{kpc}$. These additional characteristics agree with the earlier assessment that G114.3+0.3 is related to patches of $\mathrm{H}$ I at a distance of $700 \mathrm{pc}$. At this distance the SNR would be $18 \times 11 \mathrm{pc}$ in size.

At this low distance the dispersion measure and rotation measure of PSR 2334+61 become another point of debate. From the model by Taylor \& Cordes (1993) we get a foreground dispersion measure of $\mathrm{DM}_{\text {fore }}=13 \mathrm{~cm}^{-3} \mathrm{pc}$. But the pulsar has a dispersion measure of about $58 \mathrm{~cm}^{-3} \mathrm{pc}$, which would give a distance of $2.5 \mathrm{kpc}$. On the other hand we also find a very high rotation measure of $-100 \mathrm{rad} \mathrm{m}^{-2}$ which combined with the dispersion measure leads to a foreground magnetic field parallel to the line of sight of $\mathrm{B}_{\|}=-2.1 \mu \mathrm{G}$; which is significantly higher than the expected value of $\mathrm{B}_{\|}=-1 \mu \mathrm{G}$ (Mitra et al. 2003). The enhanced magnetic field indicates an additional structure along the line of sight with a strong magnetic field and a high electron density causing the additional dispersion and rotation measure. Since the pulsar is located within a supernova remnant the nature of this additional structure should be clear. Hence we now want to investigate the possibility that the shell of the expanding SNR itself causes the high RM and DM values.

The rotation measure $\mathrm{RM}$ is defined by:

$$
\frac{\mathrm{RM}}{\mathrm{B}_{\|}}=0.82 \cdot \mathrm{DM}=0.82 \cdot \int \mathrm{n}_{\mathrm{e}} \cdot \mathrm{dl},
$$

here $\mathrm{n}_{\mathrm{e}}$ represents the electron density in $\left[\mathrm{cm}^{-3}\right]$ and $\mathrm{l}$ the pathlength in $[\mathrm{pc}]$. Assuming a foreground dispersion measure of $\mathrm{DM}_{\text {fore }}=13 \mathrm{~cm}^{-3} \mathrm{pc}$ and a mean foreground magnetic field along the line of sight of $\mathrm{B}_{\|}=-1 \mu \mathrm{G}$, we get internal values of $\mathrm{DM}_{\mathrm{SNR}}=45 \mathrm{~cm}^{-3} \mathrm{pc}$, $\mathrm{RM}_{\mathrm{SNR}}=-89 \mathrm{rad} \mathrm{m}^{-2}$, and $\mathrm{B}_{\|}^{\mathrm{SNR}}=-2.4 \mu \mathrm{G}$, for the SNR.

The ratio between the internal and external magnetic field strengths results in a compression of 2.4. Assuming the same compression ratio for the interstellar medium, swept up by the expanding shockwave and a radius of $7 \mathrm{pc}$ gives a width of $1.2 \mathrm{pc}$ for the shell. Together with the internal dispersion measure we get an electron density of $38 \mathrm{~cm}^{-3}$ within the shell. Assuming the swept up material is fully ionized, a Hydrogen to Helium ratio 
of 9:1, and again a compression ratio of 2.4 yield an average ambient density of about 14 atoms $\mathrm{cm}^{-3}$. These are reasonable values and strengthen the proposed nearby location.

The surrounding patches of $\mathrm{H}$ I indicate a more homogeneous ambient medium for the supernova explosion rather than a stellar wind structured one. This would indicate a B2 or later type progenitor star - since these are not massive enough to create a strong stellar wind - and a type II supernova event.

At 700 pc distance we get a mean radius of about 7 pc for the SNR. Following Cioffi et al. (1988) a supernova remnant with an explosion energy of $\mathrm{E}_{0}\left[10^{51} \mathrm{erg}\right]$ and an ambient medium density of $\mathrm{n}_{0}\left[\mathrm{~cm}^{-3}\right]$ would enter the so-called pressure driven snowplow phase (PDS) at a radius $R_{P D S}[p c]$ defined by:

$$
\mathrm{R}_{\mathrm{PDS}}=14.0 \cdot \frac{\mathrm{E}_{0}^{2 / 7}}{\mathrm{n}_{0}^{3 / 7}},
$$

for solar abundances. For an ambient density of $\mathrm{n}_{0}=14 \mathrm{~cm}^{-3}$ and an explosion energy of

$\mathrm{E}_{0}=10^{51} \mathrm{erg}$ we derive $\mathrm{R}_{\mathrm{PDS}}=4.5 \mathrm{pc}$. This would indicate that G114.3+0.3 has already entered the PDS phase and indeed it would require an explosion energy of $\mathrm{E}_{0} \approx 5 \cdot 10^{51} \mathrm{erg}$ for the SNR to be still in the so-called energy conserving Sedov phase. With the above parameters we can calculate the mass of the swept up material to be $\mathrm{M}_{\mathrm{sw}}=480 \mathrm{M}_{\odot}$ and from the equations determined by Cioffi et al. (1988) we can also estimate an age of about 7700 yrs for G114.3+0.3.

\subsection{G116.5+1.1}

As for G114.3+0.3 we can get additional constraints on the distance from the presence of observable optical emission (Fesen et al. 1997) and polarized $1.4 \mathrm{GHz}$ radio continuum emission in the CGPS (see Fig. 4). This indicates a Local arm location, most likely closer than $2.2 \mathrm{kpc}$. The H I structure we found, surrounding the SNR, has a radial velocity of about $-17 \mathrm{~km} \mathrm{~s}^{-1}$ which translates to a location within the Local arm at about $1.6 \mathrm{kpc}$. Similar to G114.3+0.3 the surrounding H I consists of diffuse and smooth emission patches which do not display any dynamics. It looks more like the expanding shockwave of the supernova explosion created the hole, the SNR resides in, by taking away the material in the interior. Hence as for G114.3+0.3 we believe the progenitor star was a B2 or later type star and exploded in a type II event. The diffuse emission to the east in contrast to the sharp outer boundary to the west indicates that the star exploded inside this H I cloud but to the east the shockwave already left the cloud in a kind of breakout giving the SNR its smooth 
structure in that direction.

At a distance of $1.6 \mathrm{kpc}$ the SNR has a mean radius of $16.5 \mathrm{pc}$. Given the similarity in the emission structure to G114.3+0.3 we might assume that G116.5+1.1 has also just entered the PDS phase. Assuming an explosion energy of $10^{51} \mathrm{erg}$ we get with equation (2) a lower limit for the ambient density of $0.8 \mathrm{~cm}^{-3}$. This indicates that the SNR has indeed entered the PDS phase because a lower density would be quite unusual for a location within a spiral arm. Only within stellar wind bubbles we would expect a lower density but there is no evidence for such a structure around G116.5+1.1. For an ambient density of $1 \mathrm{~cm}^{-3}$ we would get an age of about $15000 \mathrm{yrs}$ and for $5 \mathrm{~cm}^{-3}$ about $50000 \mathrm{yrs}$. The mass of the material swept up by the expanding shockwave would be $450 \mathrm{M}_{\odot}$ and $2250 \mathrm{M}_{\odot}$ respectively. Since the SNR is still rather bright in radio continuum emission a smaller age implying an earlier evolutionary state is to be prefered.

\subsection{CTB $1($ G116.9+0.2)}

The associated $\mathrm{H}$ I shell of CTB 1 with a radial velocity of $-27 \mathrm{~km} \mathrm{~s}^{-1}$ indicates an interarm location. But because of the complex and highly dynamic environment of the SNR we believe that it must reside in one of the spiral arms: in the interarm one expects to have less material in which a remnant expands easier and more or less symmetrically. This makes CTB 1 either a blueshifted local or a redshifted Perseus arm object. To make a decision we have to carefully investigate the behavior and dynamics of surrounding structures in the neutral hydrogen going from CTB 1 to the local and Perseus arm (Fig. 8 and.Fig. 9) to find a connection to either of them.

Starting from low negative velocities in the local arm a smooth and large $\mathrm{H}$ I bubble forms around CTB 1, best pronounced between $-16 \mathrm{~km} \mathrm{~s}^{-1}$ and $-18 \mathrm{~km} \mathrm{~s}^{-1}$. This bubble was most likely created by stellar wind effects and/or supernova explosions a long time ago. The diffuse structure indicates a late stage of evolution in which the structure starts to merge with the surrounding medium. Towards higher negative velocities the edges of the bubble slowly transform into bright thin filaments which are moving closer to the radio bright shell of CTB 1 with decreasing velocity. Bright filamentary structures indicate a highly dynamic environment in an earlier stage of evolution where energetic interaction is still taking place. Between $-25 \mathrm{~km} \mathrm{~s}^{-1}$ and $-27 \mathrm{~km} \mathrm{~s}^{-1}$ those filaments create a bubble around CTB 1 which is closest around the radio bright shell and open to the other side where we see only smooth low

surface brightness radio emission. This structure is best pronounced at $-27 \mathrm{~km} \mathrm{~s}^{-1}$. Going from there to even higher negative velocities the structure of the bubble remains constant while it slowly fades away. At the same time smooth emission from the Perseus arm is 
starting to move in from the south. There appears to be a connection between local arm gas and the bubble around CTB 1, but not between CTB 1 and the Perseus arm. Hence CTB 1 is a blue-shifted local object.

The most likely scenario to explain the observed structures and the velocity shift is that the progenitor star of CTB 1 resided in the smooth bubble visible at local arm velocities. The star was not located in the center but in the south-west and closer to us. Thus the stellar wind and later the supernova shock wave impacted on the edges of the bubble first in the south-west giving the SNR its half shell shape. Since the progenitor of CTB 1 was located closer to us relative to the center of the bubble it also hits the edges closer to us giving it a shift in velocity since this part of the bubble is moving towards us. Hence the systemic velocity of the H I structure around CTB 1 is the same as for the smooth bubble at $-17 \mathrm{~km} \mathrm{~s}^{-1}$, assuming of course that this bubble is not shifted as well. The local arm position of CTB 1 is also supported by the presence of polarized emission, although it is very weak.

This systemic velocity translates to a distance of $1.6 \mathrm{kpc}$. We note that both SNRs G116.5+1.1 and CTB 1 appear to be located in the same large H I complex at a distance of $\sim 1.6 \mathrm{kpc}$ and the breakout region of G116.5+1.1 might even be located inside the same bubble as CTB 1. Since the structure around CTB 1 has a radial velocity of about $-27 \mathrm{~km}$ $\mathrm{s}^{-1}$ it is moving towards us at a velocity of $\sim 10 \mathrm{~km} \mathrm{~s}^{-1}$ relative to the main body of the complex. At a distance of $1.6 \mathrm{kpc}$ the linear size of the remnant would be $18 \times 15 \mathrm{pc}$.

With this interpretation, we can also understand the emission structure of the breakout region of $\mathrm{CTB} 1$ in the radio continuum map. If the remnant is located in the south-west of the bubble rather than in its center, its shockwave arrives at the lower edge of the H I bubble earlier creating the bright radio shell and giving rise to its incomplete circular shape. Hence the north-east opening of the remnant is not necessarily due to a breakout, but simply the result of a freely expanding shockwave which is traveling into the low density interior of the bubble.

\section{Summary}

We have presented new sensitive radio continuum and $\mathrm{H}$ I data at $1^{\prime}$ resolution towards

the three supernova remnants G114.3+0.3, G116.5+1.1 and CTB 1 (G116.9+0.2). The strength of the H I analysis is the combination of high resolution data with large spatial coverage. G116.5+1.1 and CTB 1 appear to be located within the same H I complex. Based on the surrounding $\mathrm{H}$ I structure and dynamics we derived new distance estimates for these 
SNRs. We also concluded that G114.3+0.3 and G116.5+1.1 are expanding inside an area not much effected by stellar wind effects of their progenitors implying supernova explosions of type II with B2 or later type progenitor stars. CTB 1, however, is expanding in a highly dynamic environment indicating an early $\mathrm{B}$ or O-type progenitor star exploding in a Ib or Ic event. We also believe that G114.3+0.3 and G116.5+1.1 are expanding inside an area not much affected by stellar wind effects of their progenitors in which case the supernova explosions would have been type II events with a B2 or later type progenitor star.

According to our analysis G114.3+0.3 is the closest remnant among the three SNRs with a distance of about $700 \mathrm{pc}$ which gives this object a mean diameter of about $14 \mathrm{pc}$. The SNR is in the so-called pressure driven snowplow phase, has swept up about $480 \mathrm{M}_{\odot}$ of material and is probably around $7700 \mathrm{yrs}$ old. The other two remnants are located at a distance of $1.6 \mathrm{kpc}$. This gives G116.5+1.1 an average diameter of $33 \mathrm{pc}$, making this SNR the biggest of the three discussed here. It has also reached the PDS phase and with typical SNR parameters we get an age more than twice as much as G114.3+0.3 and probably more than $450 \mathrm{M}_{\odot}$ of swept up material.

CTB 1 appears to be located at the edge of a large H I bubble which expands with a velocity of $\sim 10 \mathrm{~km} \mathrm{~s}^{-1}$ towards us with respect to the systemic velocity of the whole system $\left(-17 \mathrm{~km} \mathrm{~s}^{-1}\right)$. The distance of $1.6 \mathrm{kpc}$ gives the remnant a size of about $16.5 \mathrm{pc}$. We argue that the "opening" of CTB 1 in the northeast is not an effect of a breakout but simply the result of expanding into the interior of its surrounding bubble.

The Dominion Radio Astrophysical Observatory is a National Facility operated by the National Research Council. The Canadian Galactic Plane Survey is a Canadian project with international partners, and is supported by the Natural Sciences and Engineering Research Council (NSERC). We wish to thank Tom Landecker and Wolfgang Reich for careful reading of the manuscript and discussions.

\section{REFERENCES}

Brand, J., \& Blitz, L. 1993, A\&A, 275, 67

Chevalier, R. A., Kirshner, R. P., \& Raymond, J. C. 1980, ApJ, 235, 186

Becker, W., Brazier, K. T. S., \& Trümper, J. 1996, A\&A, 306, 464

Berkhuijsen, E. M. 1986, A\&A, 166, 257

Berkhuijsen, E. M. 1987, A\&A, 181, 398 
Cioffi, D. F., McKee, C. F., \& Bertschinger, E. 1988, ApJ, 334, 252

Cordes, J. M., \& Lazio, T. J. W. 2002, astro-ph/0207156

Craig, W. W., Hailey, C. J., \& Pisarski, R. L. 1997, ApJ, 488, 307

Dickel, J. R., \& Willis, A. G. 1980, A\&A, 85, 55

Fesen, R. A., Winkler, P. F., Rathore, Y., Downes, R. A., Wallace, D., \& Tweedy, R. W. 1997, AJ, 113(2), 767

Fich, M., \& Blitz, L. 1984, ApJ, 279, 125

Fich, M. 1986, ApJ, 303, 465

Frail, D. A., Kulkarni, S. R., \& Vasisht, G. 1993, Nature, 365, 136

Fürst, E., Reich, W., \& Seiradakis, J. H. 1993, A\&A, 276, 470

Green, D. A. 1984, MNRAS, 209, 449

Green, D. A., \& Gull, S. F., 1982, Nature, 299, 606

Goss, W. M., Kalberla, P. M. W., \& Schwarz, U. J., 1988, in: IAU Colloquim No. 101, Supernova Remnants and the Interstellar Medium, Cambridge University Press, Cambridge, eds. R. Roger \& T.L. Landecker, p. 239

Higgs, L. A., \& Tapping, K.F. 2000, AJ, 120, 2471

Kerr, F. J. \& Lynden-Bell, D. 1986, MNRAS, 221, 1023

Kothes, R., Uyanıker, B. \& Pineault, S. 2001, ApJ, 560, 236

Kothes, R., Uyanıker, B. \& Yar, A. 2002, ApJ, 576, 169

Kulkarni, S. R., Predehl, P. R., Hasinger, G., \& Aschenbach, B. R. 1993, Nature, 362, 135

Landecker, T. L., Roger, R. S., \& Dewdney, P. E. 1982, AJ, 87, 1379

Landecker, T. L., Dewdney, P. E., Burgess, T. A., Gray, A. D., Higgs, L. A., Hoffmann, A. P., Hovey, G. J., Karpa, D. R., Lacey, J. D., Prowse, N., Purton, C. R., Roger, R. S., Willis, A. G., Wyslouzil, W., Routledge, D., \& Vaneldik, J. F. 2000, A\&AS, 145, 509

Lozinskaya, T. A. 1980, A\&A, 84, 26

Mavromatakis, F., Boumis, P., \& Paleologou, E. V. 2002, A\&A, 383, 1011 
Mitra, D., Wielebinski, R., Kramer, M., \& Jessner, A. 2003, A\&A, 398, 993

Pineault, S., Landecker, T. L., Madore, B., \& Gaumont-Guay, S. 1993, AJ, 105, 1060

Reed, J. E., Hester, J. J., Fabian, A. C., \& Winkler, P. F. 1995, ApJ, 440, 706

Reich, W., \& Braunsfurth, E. 1981, A\&A, 99, 71

Reynoso, E. M., Velázquez, P. F., Dubner, G. M., \& Goss, W. M. 1999, AJ, 117, 1827

Routledge, D., Dewdney, P. E., Landecker, T. L., \& Vaneldik, J. F. 1991, A\&A, 247, 529

Taylor, A. R., Gibson, S. J., Peracaula, M., Martin, P. G., Landecker, T. L., Brunt, C. M., Dewdney, P. E., Dougherty, S. M., Gray, A. D., Higgs, L. A., Kerton, C. R., Knee, L. B. G., Kothes, R., Purton, C. R.; Uyanıker, B., Wallace, B. J., Willis, A. G. \& Durand, D 2003, AJ, 125, 3145

Taylor, J. H., \& Cordes, J. M. 1993, ApJ, 411, 674

Uyanıker, B., Landecker, T. L., Gray, A. D., \& Kothes, R. 2003, ApJ, 585, 785

van den Berg, S., Marscher, A. P., \& Terzian, Y. 1973, ApJS, 26, 19

Wallace, B. J., Landecker, T. L. \& Taylor, A. R. 1994, A\&A, 286, 565

Westerhout, G., \& Wendlandt, H.-U. 1982, A\&AS, 49, 143

Willis, A. G. 1973, A\&A, 26, 237

Willis, A. G. 1999, A\&AS, 136, 603

Yar, A., Uyanıker, B., \& Kothes, R. 2002, in: ASP conf. series, Vol. 276, p. 351, eds. A. R. Taylor, T. L. Landecker \& A. G. Willis 
Fig. 1. - Radio continuum image of the three supernova remnants, CTB 1 (G116.9+0.2), G116.5+1.1 and G114.3+0.3 from the CGPS at $1420 \mathrm{MHz}$.

Fig. 2.- Total intensity map of G114.3+0.3 at $21 \mathrm{~cm}$ radio continuum. Contours start at $5.5 \mathrm{~K} \mathrm{~T}_{\mathrm{B}}$ total intensity up to $7.4 \mathrm{~K} \mathrm{~T} \mathrm{~T}_{\mathrm{B}}$ in steps of $0.16 \mathrm{~K} \mathrm{~T}_{\mathrm{B}}$. The position of the PSR2334+61 is indicated with a star. Towards G114.3+0.3 there are three H II regions, $\mathrm{S} 163\left(\ell=113.5^{\circ}, b=-0.6^{\circ}\right), \mathrm{S} 165\left(\ell=114.6^{\circ}, b=+0.2^{\circ}\right)$, and S166 $\left(\ell=114.6^{\circ}, b=-0.8^{\circ}\right)$.

Fig. 3.- Contour map of CTB 1 and G116.5+1.1 at $21 \mathrm{~cm}$ radio continuum. Contours represent total intensity starting at $5.5 \mathrm{~K} \mathrm{~T}_{\mathrm{B}}$ in steps of $0.12 \mathrm{~K} \mathrm{~T}_{\mathrm{B}}$ up to $6.7 \mathrm{~K}_{\mathrm{B}}$, in black. The higher emission regions are represented by white contours starting at $7 \mathrm{~K} \mathrm{~T}_{\mathrm{B}}$ and 7.5 to $10.5 \mathrm{~K} \mathrm{~T}_{B}$ in steps of $1.0 \mathrm{~K} \mathrm{~T}_{\mathrm{B}}$.

Fig. 4.- Polarized intensity images towards the three SNRs at 2 arcminute resolution. Gray scale extends from 0.02 (white) to $0.15 \mathrm{~K} \mathrm{~T}_{\mathrm{B}}$ (black). Contours start from $0.1 \mathrm{~K} \mathrm{~T}_{\mathrm{B}}$ and run in steps of $0.025 \mathrm{~K} \mathrm{~T}_{\mathrm{B}}$. White contours are overlaid to show the total intensity emission.

Fig. 5.- Averaged H I map towards the supernova remnant G114.3+0.3. The H I line emission is averaged between $v_{\mathrm{LSR}}=-4.8 \mathrm{~km} \mathrm{~s}^{-1}$ and $v_{\mathrm{LSR}}=-7.2 \mathrm{~km} \mathrm{~s}^{-1}$. The $\mathrm{H} \mathrm{I}$ intensity contours (in black) start from $40 \mathrm{~K} \mathrm{~T}_{\mathrm{B}}$ up to $80 \mathrm{~K}_{\mathrm{B}}$ with $10 \mathrm{~K} \mathrm{~T}_{\mathrm{B}}$ increasing steps. The $21 \mathrm{~cm}$ radio continuum emission is represented by white contours at from $6.2 \mathrm{~K}$ $\mathrm{T}_{\mathrm{B}}$ and $6.6 \mathrm{~K} \mathrm{~T}_{\mathrm{B}}$. The noise in the maps is $1.7 \mathrm{~K} \mathrm{~T}_{\mathrm{B}}$, due to averaging of images.

Fig. 6.- Left: Averaged H I maps towards the SNRs CTB 1 and G116.5+1.1. The left-hand panel shows the H I line emission averaged over velocity channels between $v_{\text {LSR }}=-15.5 \mathrm{~km}$ $\mathrm{s}^{-1}$ and $v_{\mathrm{LSR}}=-18.5 \mathrm{~km} \mathrm{~s}^{-1}$. The $\mathrm{H}$ I contours (in black) start from $10 \mathrm{~K} \mathrm{~T}_{\mathrm{B}}$ and run to 38 $\mathrm{K} \mathrm{T}_{\mathrm{B}}$, in $7.0 \mathrm{~K} \mathrm{~T}$ B steps. Right: $\mathrm{H} \mathrm{I}$ intensities averaged from $v_{\mathrm{LSR}}=-27.5$ to $v_{\mathrm{LSR}}=-30.5$ $\mathrm{km} \mathrm{s}^{-1}$. Contour levels are 6 to $46 \mathrm{~K} \mathrm{~T}_{\mathrm{B}}$ with steps of $10 \mathrm{~K} \mathrm{~T}_{\mathrm{B}}$. The $21 \mathrm{~cm}$ radio continuum emission, in both images, is overlaid as white contours at 6.0 and $7.0 \mathrm{~K} \mathrm{~T}_{\mathrm{B}}$. The noise in the maps is $1.5 \mathrm{~K} \mathrm{~T}_{\mathrm{B}}$, due to averaging of images over $3 \mathrm{~km} \mathrm{~s}^{-1}$. 
Fig. 7.- The radial velocity of SNRs and H II regions within $20^{\circ}$ in Galactic longitude of CTB 1 as a function of distance. The radial velocities of our three target supernova remnants are indicated. Information about the H II regions were taken from Brand \& Blitz (1993). The radial velocities and distances of the SNRs were taken from: Kothes et al. (2001) (G106.3+2.7), Kothes et al. (2002) (CTB 109), Goss et al. (1988)\& Reed et al. (1995) (Cas A), Pineault et al. (1993) (CTA 1), Reynoso et al. (1999) \& Chevalier et al. (1980) (Tycho), Yar-Uyanıker et al. (2004; in preparation) (G126.2+1.6 and G127.2+0.5), Green \& Gull (1982) \& Wallace et al. (1994) (3C58), and Routledge et al. (1991) (HB 3).

Fig. 8. - Distribution of the H I emission observed towards the supernova remnants CTB 1 and G116.5+1.1, as channel maps. The radio continuum emission from CTB 1 shown by a contour at $7 \mathrm{~K} \mathrm{~T}_{\mathrm{B}}$ to indicate the position of the SNR on the $\mathrm{H}$ I channel maps.

Fig. 9.- Same as Fig. 8 but for different velocity intervals as labeled on the panels. 
This figure "f1.jpg" is available in "jpg" format from: http://arxiv.org/ps/astro-ph/0408386v1 
This figure "f2.jpg" is available in "jpg" format from: http://arxiv.org/ps/astro-ph/0408386v1 
This figure "f3.jpg" is available in "jpg" format from: http://arxiv.org/ps/astro-ph/0408386v1 
This figure "f4.jpg" is available in "jpg" format from: http://arxiv.org/ps/astro-ph/0408386v1 
This figure "f5.jpg" is available in "jpg" format from: http://arxiv.org/ps/astro-ph/0408386v1 
This figure "f6.jpg" is available in "jpg" format from: http://arxiv.org/ps/astro-ph/0408386v1 
This figure "f7.jpg" is available in "jpg" format from: http://arxiv.org/ps/astro-ph/0408386v1 
This figure "f8.jpg" is available in "jpg" format from: http://arxiv.org/ps/astro-ph/0408386v1 
This figure "f9.jpg" is available in "jpg" format from: http://arxiv.org/ps/astro-ph/0408386v1 